\title{
Parametrically Stimulated Recovery of a Microwave Signal Stored in Standing Spin-Wave Modes of a Magnetic Film
}

\author{
A. A. Serga, ${ }^{1}$ A. V. Chumak, ${ }^{1,2}$ A. André, ${ }^{1}$ G. A. Melkov, ${ }^{2}$ A. N. Slavin, ${ }^{3}$ S. O. Demokritov, ${ }^{4}$ and B. Hillebrands ${ }^{1}$ \\ ${ }^{1}$ Fachbereich Physik and Forschungsschwerpunkt MINAS, Technische Universität Kaiserslautern, 67663 Kaiserslautern, Germany \\ ${ }^{2}$ Department of Radiophysics, Taras Shevchenko National University of Kiev, Kiev, Ukraine \\ ${ }^{3}$ Department of Physics, Oakland University, Rochester, Michigan 48309, USA \\ ${ }^{4}$ Institut für Angewandte Physik, Westfälische Wilhelms-Universität Münster, Münster, Germany
}

(Received 13 September 2007; published 27 November 2007)

\begin{abstract}
We demonstrate that a microwave signal carried by a packet of dipolar spin waves propagating in a tangentially magnetized magnetic film can be stored in the form of standing dipole-exchange spin-wave modes of the film and can be recovered by means of a double-frequency parametric pumping mechanism. This mechanism is based on the parametric amplification of the standing (thickness) modes of the film by external pumping. The time of recovery, duration, and power of the recovered pulse signal are controlled by the power of the pumping signal.
\end{abstract}

DOI: $10.1103 /$ PhysRevLett.99.227202

PACS numbers: 75.30.Ds, 76.50.+g, 85.70.Ge

The challenge to store and process information using an ensemble of natural excitations of matter has been intensively addressed both theoretically and experimentally. For a long time, the search has concentrated on different types of echo-based phenomena involving phase conjugation techniques [1]. Several years ago, in the field of spinwave propagation, a different method of microwave signal recovery was proposed and tested experimentally [2]. A dipolar spin-wave signal was scattered on random impurities and defects of a ferrimagnetic medium, and restoration was performed by frequency-selective parametric amplification of a narrow band of scattered waves having frequencies close to the carrier frequency of the original signal wave. Although this method is rather effective, the restored microwave signal is noiselike and only partially coherent, as it is formed by many waves having close but arbitrarily shifted phases [3].

In this Letter, we report on novel experiments demonstrating storage and recovery of a microwave signal. In contrast with all of the previous work, where continuous spectra of natural excitations of matter were utilized for signal storage and recovery, in our current Letter we report experiments where storage and recovery of a microwave signal were performed using a single standing spin-wave mode which belongs to the discrete dipole-exchange spinwave spectrum of a magnetic film. The standing spin-wave modes exist in magnetic films due to the spatial confinement of the magnon gas caused by the finite film thickness. In this case, the restored signal has a practically noiseless character, as it is formed by a single thickness spin-wave mode of the film.

Our experiments were performed in a ferrimagnetic epitaxial yttrium iron garnet (YIG) film. The process of signal storage was happening naturally by means of excitation of a long-lifetime standing spin-wave mode by a signal wave propagating in the film, as discussed below. In contrast, for the signal recovery it was necessary to apply to the film an external pumping microwave field with the frequency that is exactly twice as large as the carrier frequency of the stored signal.

The setup used in our experiments is shown in Fig. 1. The input electromagnetic microwave pulse was converted by the input microstrip transducer into a pulse of dipolar spin waves that propagate in a long and narrow $(30 \times$ $1 \mathrm{~mm}^{2}$ ), $5 \mu \mathrm{m}$ thick monocrystalline YIG waveguide (saturation magnetization $4 \pi M_{s}=1750 \mathrm{G}$, and the exchange constant is $D=5.4 \times 10^{-9} \mathrm{Oe} \cdot \mathrm{cm}^{2}$ ). The output transducer, used to receive the output microwave signal, was placed at a distance of $l=8 \mathrm{~mm}$ away from the input transducer. A bias magnetic field of $H_{0}=1716$ Oe was applied in the plane of the YIG waveguide, along its width and perpendicular to the direction of spin-wave propagation. At the input transducer, rectangular microwave pulses of 100 ns duration and a carrier frequency $f_{\text {in }}=$ 7.040 GHz were applied. These pulses excite wave packets of so-called magnetostatic surface spin waves (MSSWs) (or Damon-Eshbach magnetostatic waves [4]), having a carrier wave number $k_{\text {in }} \simeq 100 \mathrm{~cm}^{-1}$. The group velocity $v_{g}$ of the excited wave packet is about $2.3 \mathrm{~cm} / \mu \mathrm{s}$. This

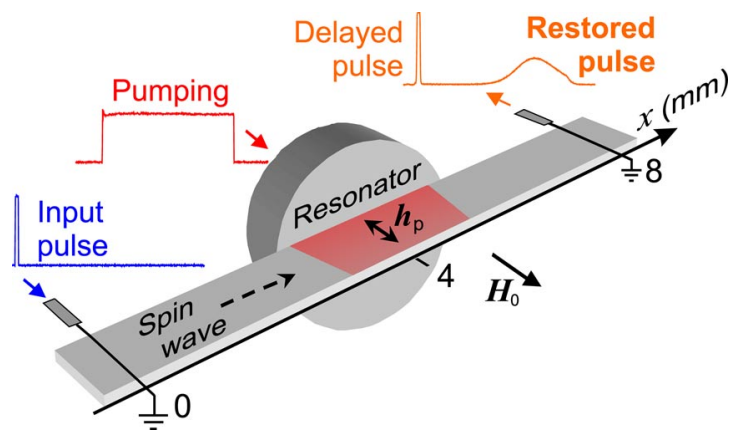

FIG. 1 (color online). Experimental setup and typical waveforms of the input, output, and pumping pulses. 
group velocity determines the time delay of the propagating wave packet between the input and output transducers of about $350 \mathrm{~ns}$. The spin-wave packet received by the output transducer is again converted into an electromagnetic pulse which is observed using an oscilloscope.

An open dielectric resonator placed in the middle of the waveguide and excited by the external microwave source produces a pumping magnetic field $\vec{h}_{p}$ that is parallel to the static bias magnetic field $\vec{H}_{0}$; see Fig. 1. Its resonance frequency $f_{r}=14.078 \mathrm{GHz}$ was chosen to be close to twice the carrier frequency of the input microwave pulse. Thus, the condition for so-called parametric parallel pumping [5] is fulfilled. In this case, the energy of the pumping field is most effectively transferred to magnetic oscillations and waves having a frequency that is exactly half of the pumping frequency.

The experimental procedure is illustrated by the waveforms shown in Fig. 1. The left one shows the time profile of the input microwave signal applied to the input transducer. The middle one illustrates the profile of the doublefrequency pumping field applied to the dielectric resonator. The right waveform shows the time profile of the output signal consisting of two pulses. The first one is the delayed pulse caused by the travelling MSSW packet, excited by the input signal. The delay time of this pulse is determined by the distance between the transducers and the group velocity of the spin-wave packet. The second, broader pulse appears only after the application of the parametric pumping. It arrives at the output transducer substantially later than the delayed pulse. Separate measurements (not shown here) using an additional output transducer, placed $1 \mathrm{~mm}$ apart from the main one, have shown that the group velocity of this additional spin-wave packet coincides with that of the first delayed spin-wave packet. Thus, the experimental data presented in Fig. 1 suggest that the second delayed pulse is caused by the spin-wave packet released by the parametric pumping from spin-wave modes, excited by the initial propagating packet in the area close to the pumping resonator and existing for a certain time defined by the (rather long) lifetime of standing spin waves after the initial (signal) wave packet has passed this area.

We would like to emphasize that the second, delayed pulse is not a product of a direct parametric amplification of the propagating input spin-wave packet, since it is even found if the pumping field is switched on after the input spin-wave packet has passed the resonator. Moreover, at a given carrier frequency of the input microwave pulse, the second (recovered) pulse is observed only if the halffrequency of the microwave pumping lies inside the narrow frequency intervals close to the positions of the dipoleexchange "gaps" in the frequency spectrum of the magnetic film (see, e.g., Chapter 7 in Ref. [5] as well as [6]). The observation of the restored spin-wave packets in these narrow frequency intervals suggests that the process of storage and restoration of the initial microwave signal is connected to the discrete thickness-related exchangedominated standing spin-wave modes of the magnetic film that form the dipole-exchange gaps in the spectrum due to their hybridization with the lowest dipoledominated propagating spin-wave mode in the film [6].

To understand the mechanism governing the observed storage-and-recovery effect, let us consider the dipoleexchange spectrum in MSSW geometry with in-plane carrier wave number $k_{x}$ in a film of thickness $L$. A calculated spectrum is shown in Fig. 2(b). It consists of a DamonEshbach-type spin wave with finite slope (nonzero group velocity) [4] crossing several dispersionless standing spinwave modes indicated in Fig. 2(b) by the corresponding mode index $n$. These modes travel perpendicular to the film, and, assuming the simple case of unpinned surface spins, the wave vector can be written as $k_{\perp}=\pi n / L$, where $n=1,2,3, \ldots$. In the crossing regimes, a small mode repulsion is obtained, and so-called "dipoleexchange gaps" in the spin-wave spectrum are formed (see, e.g., [6] for details). Under the experimental conditions used in the work presented here, the frequency separation between the discrete spin-wave modes of the film is $10-20 \mathrm{MHz}$, depending on the mode number $n$.

Since the frequency spectrum of a short (duration $100 \mathrm{~ns})$ propagating spin-wave packet is several times wider than the frequency separation between two neighboring discrete standing spin-wave modes, this packet can excite standing modes during its course of propagation. Because of their nearly zero group velocity, these modes do not propagate away from the point where they are excited. Instead they form a "trail" of the MSSW pulse which exists for several microseconds, reminiscent to a contrail of a flying airplane.

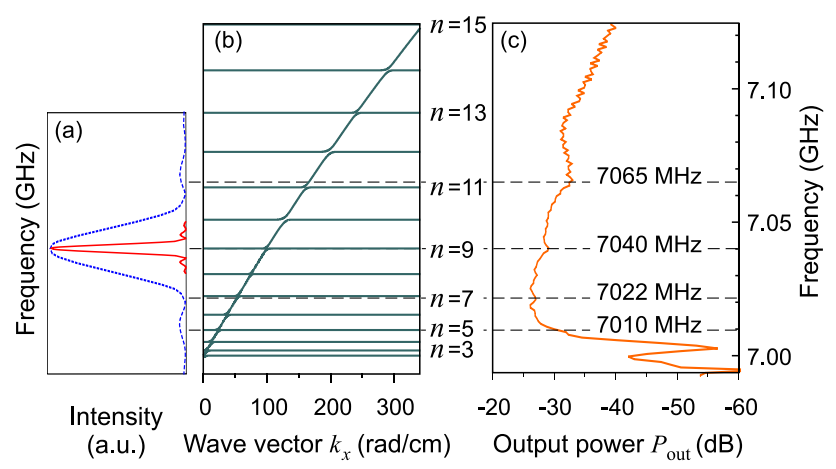

FIG. 2 (color online). (a) Frequency spectra of the input pulse (dotted blue line) and pumping pulse (transferred to half of the carrier pumping frequency) (solid red line). (b) Calculated dipole-exchange spectrum of traveling spin waves in the experimental YIG film ( $n$ is the number of a corresponding thickness mode). (c) Transmission-loss characteristics of the experimental section. The horizontal dashed lines mark the frequencies at which the recovered microwave pulse was observed when the double-frequency pumping was applied. 
In contrast, a long pumping pulse (duration of a few microseconds), which has a relatively narrow frequency spectrum, will amplify only one of the excited standing modes. The parametrically amplified standing mode reemits a traveling MSSW packet exactly in the same manner as this mode was previously excited by the signal MSSW. Thus, the process of generation of the recovered pulse can be understood as a restoration of the initial signal that was dynamically stored in the magnetic film in the form of an discrete standing spin wave.

It is necessary to note that, although the parametrically recovered pulse is directly related to the input spin-wave packet and never appears without previous application of the input signal, its characteristics, such as peak power, duration, and delay time, are determined mainly by the process of parametric interaction with pumping. This fact is illustrated by Fig. 3, where the profiles of the restored pulses are presented for different values of the pumping power. Experimental dependencies of the peak power $P_{r}$, recovery time $t_{r}$, and duration $\Delta t_{r}$ of the restored pulse on the pumping power $P_{p}$ are shown in Fig. 4 by the symbols. It is evident that an increase of the pumping power $P_{p}$ leads to a decrease of $t_{r}$ and $\Delta t_{r}$ and to an increase of $P_{r}$.

In order to understand the experimental dependencies of the temporal and amplitude characteristics of the recovered microwave pulse on the pumping power, we use the general theory of parametric interaction of spin waves (socalled "S theory") [7]. According to this theory, two concurring processes may take place simultaneously: (i) amplification of the signal spin wave and (ii) amplification of spin-wave modes from the thermal floor. Pumping with a sufficiently large amplitude $h_{p}>h_{\mathrm{th}}=$ $\Gamma_{k} / V_{k}$ (where $\Gamma_{k}$ is the relaxation frequency of a spin wave with wave number $k$ and $V_{k}$ is the coefficient of parametric coupling between this wave and the pumping field $h_{p}$ ) will parametrically generate spin waves from the thermal floor

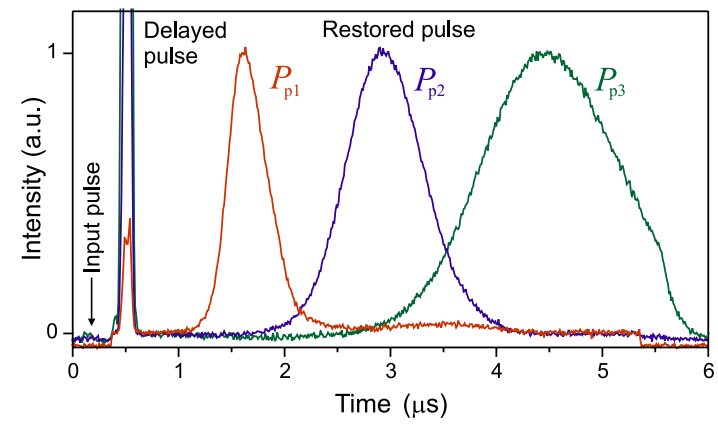

FIG. 3 (color online). Waveforms of the delayed and recovered pulses for different values of the pumping power $P_{p}: P_{p 1}=$ $1.28 \mathrm{~W}, P_{p 2}=0.52 \mathrm{~W}, P_{p 3}=0.34 \mathrm{~W}$. Waveforms are normalized by the maximum intensity of each of the recovered pulses. The carrier frequency and power of the input pulse are $7040 \mathrm{MHz}$ and $P_{\text {in }}=10 \mu \mathrm{W}$, respectively (see Fig. 2). The low pedestal under the delayed and recovered pulses corresponds to the pumping pulse. whose amplitudes will increase with time $t$ as $\exp \left(h_{p} V_{k}-\right.$ $\left.\Gamma_{k}\right) t$ (see, e.g., [5]). Moreover, it follows from Ref. [7] that, even in the case when the pumping amplitude exceeds the threshold of parametric generation for several different spin-wave groups of spin-wave modes, only modes in one of these groups, having the maximum gain factor of parametric amplification $h_{p} V_{k}-\Gamma_{k}$, will be excited. In our experimental geometry, this dominating group is formed by short exchange-dominated spin waves having wave numbers $k \simeq 10^{5} \mathrm{rad} / \mathrm{cm}$ and propagating in the film plane perpendicularly to the direction of magnetization of the sample. All of the other spin waves experience combined and competing influence of the external pumping field and of an additional internal pumping field created by the above-mentioned dominating spin-wave group. The internal ("spin-wave") pumping process almost compensates the influence of the external pumping on the other spin waves in the sample, and their amplitudes remain close to the thermal level. As one result, no amplification of standing spin waves in dipole-exchange gaps is obtained if no input signal is applied.

In contrast, when an input signal is applied, it leaves behind a trail of linearly excited standing spin-wave modes existing near the dipole-exchange gaps in the spectrum of the film (see Fig. 2) and having amplitudes $A_{s 0}$ significantly exceeding the thermal level. Thus, when the pumping signal is applied, it starts to amplify both the highamplitude standing spin waves in the spectral gaps and the

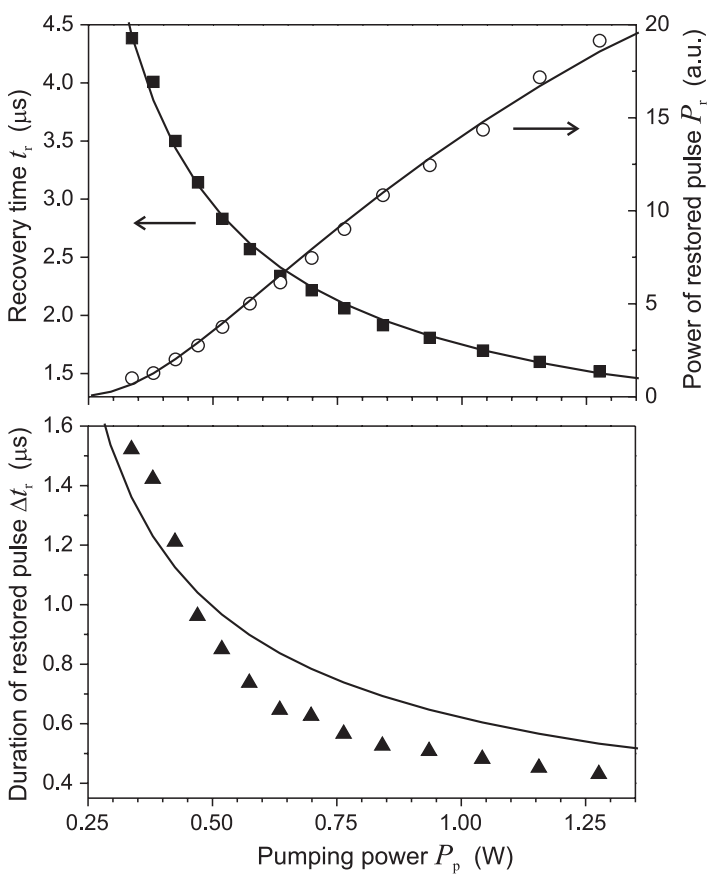

FIG. 4. Delay time (squares), peak power (open circles), and duration (triangles) of the restored pulse as functions of the pumping power. Solid lines: Results of calculation using Eqs. (1)-(3), respectively. 
short-wavelength exchange spin waves from the dominating group, whose amplitudes arise from the thermal level $A_{T} \ll A_{s 0}$. It is obvious that the amplitude of the recovered microwave pulse, formed from the amplified standing waves, will reach the maximum value $A_{s \max }$ at the moment of time $t_{\max }$ when the amplitude of the dominating spinwave group reaches a certain critical value $A_{k c r}$ which is sufficient to stop the parametric amplification of the standing modes:

$$
t_{\max }=\frac{1}{h_{p} V_{k}-\Gamma_{k}} \ln \left(\frac{A_{k \mathrm{cr}}}{A_{T}}\right) .
$$

Thus, in the stationary regime $t>t_{\max }$, standing spin waves will be suppressed, and the maximum amplitude of these waves at $t=t_{\max }$ can be written as

$$
A_{s \max }=A_{s 0} e^{\left(h_{p} V_{s}-\Gamma_{s}\right) t_{\max }}=A_{s 0}\left(\frac{A_{k \mathrm{cr}}}{A_{T}}\right)^{\left(h_{p} V_{s}-\Gamma_{s}\right) /\left(h_{p} V_{k}-\Gamma_{k}\right)},
$$

where $\Gamma_{s}$ and $V_{s}$ are the relaxation frequency of standing modes and their coefficient of parametric coupling with pumping, respectively. The solid lines in Fig. 4 were calculated by using Eqs. (1) and (2) and assuming that $P_{r}=A_{s \max }^{2}$. In this calculation, we also assumed that $\ln \left(A_{k \mathrm{cr}} / A_{T}\right)=10.25$ and that the widths of the resonant curves of the dominating spin-wave group and the standing modes were $\Delta H_{k}=2 \Gamma_{k} / \gamma=0.42 \mathrm{Oe}$ and $\Delta H_{s}=$ $2 \Gamma_{s} / \gamma=0.485 \mathrm{Oe}$, respectively, using a gyromagnetic ratio of $\gamma=2.8 \mathrm{MHz} / \mathrm{Oe}$.

The duration of the recovered pulse (see Fig. 4) can be theoretically estimated taking into account the frequencyselective character of the parametric amplification process. Because of this selectivity, the frequency bandwidth of the parametrically amplified spin waves (and, therefore, the duration of the restored pulse formed by these spin waves) is determined by the intensity and duration of the pumping pulse. In the approximation of strong pumping, the restored pulse has a Gaussian profile with a duration $\Delta T_{r}$ at half the maximum level [8]:

$$
\Delta T_{r}=2 \sqrt{\frac{t_{\max } \ln 2}{h_{p} V_{k}}}=2 \sqrt{\frac{\ln \left(A_{k \mathrm{cr}} / A_{T}\right) \ln 2}{\left(h_{p} V_{k}-\Gamma_{k}\right) h_{p} V_{k}}} .
$$

We assume here that the duration of the parametric amplification process for the standing modes is limited by the influence of the dominating group of exchange spin waves, and, therefore, the effective pumping duration can be estimated as $t_{\max }$. From Fig. 4, one can see that Eq. (3) gives a good description of the experimental data. We believe that an even better description of the experimental results could be achieved if experimental parameters such as the finite duration of the input signal, realistic widths of the dipoleexchange gaps, and nonlinear properties of the standing spin-wave modes are accounted for in the theory.

In conclusion, we observed the effect of coherent storage and recovery of a microwave signal in a ferrite film. The signal is stored in the film in the form of standing spin waves excited by the input microwave pulse in the frequency intervals near the dipole-exchange gaps, i.e., near the points of hybridization between the lowest (dipoledominated) spin-wave mode of the film and the higherorder (exchange-dominated) spin-wave modes. The signal is recovered by a long and powerful pumping pulse having the carrier frequency close to double the central frequency of one of the spectral gaps. The proposed simple quasilinear theory of parametric amplification by nonstationary (pulsed) pumping provides a good qualitative explanation of the experimental results presented in Figs. 3 and 4. These results might pave the way to new approaches of signal processing using combined microwave/spin-wave devices.

This work was partially supported by the Deutsche Forschungsgemeinschaft (No. SFB/TRR 49 and No. DE 639/6), by the Ukrainian Fund for Fundamental Research (No. 25.2/009), by MURI Grant No. W911NF-04-1-0247 from the U.S. Army Research Office, and by the Oakland University Foundation.

[1] A. Korpel and M. Chatterjee, Proc. IEEE 69, 1539 (1981).

[2] G. A. Melkov, Yu. V. Kobljanskyj, A.A. Serga, V.S. Tiberkevich, and A. N. Slavin, Phys. Rev. Lett. 86, 4918 (2001).

[3] Yu. V. Kobljanskyj, V. S. Tiberkevich, A. V. Chumak, V. I. Vasyuchka, G. A. Melkov, and A.N. Slavin, J. Magn. Magn. Mater. 272, 991 (2004).

[4] R. W. Damon and J. R. Eshbach, J. Phys. Chem. Solids 19, 308 (1961).

[5] A. G. Gurevich and G. A. Melkov, Magnetization Oscillations and Waves (CRC Press, New York, 1996).

[6] B. A. Kalinikos and A. N. Slavin, J. Phys. C 19, 7013 (1986).

[7] V.S. L'vov, Wave Turbulence under Parametric Excitations. Applications to Magnetics (Springer-Verlag, Berlin, 1994).

[8] G. A. Melkov, A. A. Serga, V.S. Tiberkevich, A.N. Oliynyk, A. V. Bagada, and A. N. Slavin, J. Exp. Theor. Phys. 89, 1189 (1999). 